\title{
A NOTE ON THE INHERITANCE OF PROPERTIES OF LOCALLY CONVEX SPACES BY SUBSPACES OF COUNTABLE CODIMENSION
}

\author{
MARK LEVIN AND STEPHEN SAXON
}

ABSTRACT. A locally convex space $E$ is said to be $\omega$-barrelled if every countable weak* bounded subset of its topological dual $E^{\prime}$ is equicontinuous; to have property $(\mathrm{C})$ if every weak* bounded subset of $E^{\prime}$ is relatively weak* compact; to have property $(\mathrm{S})$ if $E^{\prime}$ is weak* sequentially complete. If a locally convex space possesses any of the above properties, then so do all of its linear subspaces of countable codimension. Examples are furnished to show that the mentioned properties are distinct from each other.

In [4], the authors proved that a linear subspace of countable codimension in a barrelled space is barrelled. In this paper, we see that the properties of a space being $\omega$-barrelled and having weak*sequentially complete dual are inherited by subspaces of countable codimension. Only a partial result is obtained concerning the seemingly most important property, that of being a Mackey space. The paper concludes with examples to demonstrate that the various concepts discussed are, in fact, disjoint.

1. The notation will be essentially that used by J. Horváth [3]. If $\langle E, F\rangle$ is a dual pairing ( $E$ and $F$ not necessarily separating points), then $\sigma(E, F)$ will denote the topology on $E$ of pointwise convergence on $F$. The polar $A^{\circ}$ of a subset $A$ of $E$ is the set $\{f \in F:|\langle a, f\rangle| \leqq 1$ for all $a \in A\} . \tau(F, E)$ will denote the topology on $F$ of uniform convergence on $\sigma(E, F)$-compact subsets of $E$. The vector space of continuous linear functionals on a locally convex space $E$ will be designated $E^{\prime}$. A (not necessarily Hausdorff) locally convex space $E$ is said to be barrelled if every closed, balanced, convex, absorbing subset of $E$ is a neighborhood of 0 or, equivalently, if every $\sigma\left(E^{\prime}, E\right)$-bounded subset of $E^{\prime}$ is equicontinuous. A locally convex space $E$ is said to be $\omega$-barrelled if every countable, $\sigma\left(E^{\prime}, E\right)$-bounded subset of $E^{\prime}$ is equicontinuous; to have property $(\mathrm{C})$ if every $\sigma\left(E^{\prime}, E\right)$-bounded subset of $E^{\prime}$ is relatively $\sigma\left(E^{\prime}, E\right)$-countably compact; to have property (S) if $E^{\prime}$ is $\sigma\left(E^{\prime}, E\right)$-sequentially complete; to be a Mackey space if it has the topology $\tau\left(E, E^{\prime}\right)$. The codimension of a linear subspace $M$ of

Presented to the Society, January 24, 1969; received by the editors June 10, 1970. AMS 1970 subject classifications. Primary 46A05, 46A15.

Key words and phrases. Barrelled space, Mackey space, locally convex space, $\omega$-barrelled space, countable codimension.

Copyright @ 1971, American Mathematical Society 
vector space $E$ is the (algebraic) dimension of the quotient space $E / M$. $\omega$ will stand for the set of natural numbers.

2. We first state the following consequence of the main lemma of $[4]$ :

Lemma. Let E be a locally convex space with property (S). Let $M$ be a dense linear subspace of countable codimension in $E$. If a subset $B$ of $E^{\prime}$ is $\sigma\left(E^{\prime}, M\right)$-bounded, then $B$ is $\sigma\left(E^{\prime}, E\right)$-bounded.

Proof. The polar $B^{\circ}$ of $B$ in $E$ is a balanced, convex, closed subset of $E$. Since $B$ is $\sigma\left(E^{\prime}, M\right)$-bounded, it is true that $M \subset \operatorname{sp}\left(B^{\circ}\right)$. By Lemma 3 of [4], $\operatorname{sp}\left(B^{\circ}\right)$ is closed. Since $M$ is dense in $E, \operatorname{sp}\left(B^{\circ}\right)$ consists of all of $E$. Then $B$ is $\sigma\left(E^{\prime}, E\right)$-bounded.

Our first step to the desired result is the

Proposition. Let $M$ be a dense linear subspace of countable codimension in a locally convex space $E$. If $E$ is $\omega$-barrelled, has property $(\mathrm{C})$, or has property (S), then $M$ has the corresponding property when furnished with the relative topology.

Proof. $M^{\prime}$ can be canonically identified with $E^{\prime}$. Suppose $E$ is $\omega$-barrelled. Let $B$ be any countable $\sigma\left(E^{\prime}, M\right)$-bounded subset of $E^{\prime}$. By the lemma, $B$ is $\sigma\left(E^{\prime}, E\right)$-bounded. Since $E$ is $\omega$-barrelled, $B$ is equicontinuous with respect to $E$, thus $B$ is equicontinuous with respect to $M$. Then $M$ is $\omega$-barrelled.

Now suppose $E$ has property (C). Let $B$ be any $\sigma\left(E^{\prime}, M\right)$-bounded infinite subset of $E^{\prime}$. By the lemma, $B$ must also be $\sigma\left(E^{\prime}, E\right)$-bounded. Then $B$ has a $\sigma\left(E^{\prime}, E\right)$-accumulation point $f \in E^{\prime} . f$ must then be a $\sigma\left(E^{\prime}, M\right)$-accumulation point of $B$. Therefore $M$ has property (C).

Finally, suppose $E$ has property $(\mathrm{S})$. Let $\left\{f_{n}\right\}_{n \in \omega}$ be a $\sigma\left(E^{\prime}, M\right)$ Cauchy sequence in $E^{\prime}$. The set $\left\{f_{n}: n \in \omega\right\}$ must be $\sigma\left(E^{\prime}, M\right)$ bounded, and by the lemma, $\sigma\left(E^{\prime}, E\right)$-bounded. Let $X$ be a countable subset of $E$ such that $M \cup X$ spans $E$ (algebraically). Then $\left\{f_{n}(x)\right\}_{n \in \omega}$ is a bounded sequence of scalars for each $x \in X$. By diagonalization, a subsequence $\left\{g_{k}\right\}_{k \in \omega}$ of $\left\{f_{n}\right\}_{n \in \omega}$ can be obtained such that $\left\{g_{k}\right\}_{k \in \omega}$ is $\sigma\left(E^{\prime}, E\right)$-Cauchy. The subsequence therefore converges in $\sigma\left(E^{\prime}, E\right)$ to some $f \in E^{\prime}$. Since $\left\{f_{n}\right\}_{n \in \omega}$ is $\sigma\left(E^{\prime}, M\right)$-Cauchy, it also converges in $\sigma\left(E^{\prime}, M\right)$ to $f$. Then $M$ has property (S).

3. We now turn our attention to closed subspaces of countable codimension.

Proposition. Let $M$ be a closed linear subspace of countable codimension in a locally convex space $E$. If $E$ is w-barrelled, has property (C), 
or has property (S), then $M$ has the corresponding property when furnished with the relative topology.

Proof. Let $P$ be any algebraic projection of $E$ onto $M$. Suppose $E$ has property (S). Let $\left\{f_{n}\right\}_{n \in \omega}$ be a $\sigma\left(M^{\prime}, M\right)$-Cauchy sequence in $M^{\prime}$. By Lemma 2 of [4], the sequence $\left\{f_{n} \circ P\right\}_{n \in \omega}$ is in $E^{\prime}$. It is easily seen that $\left\{f_{n} \circ P\right\}_{n \in \omega}$ is $\sigma\left(E^{\prime}, E\right)$-Cauchy. There must exist $g \in E^{\prime}$ such that $\left\{f_{n} \circ P\right\}_{n \in \omega}$ converges to $g$ in $\sigma\left(E^{\prime}, E\right)$. Let $f$ be the restriction of $g$ to $M$. Then $\left\{f_{n}\right\}_{n \in \omega}$ converges to $f$ in $\sigma\left(M^{\prime}, M\right)$. Then $M$ has property (S). The other assertions of the proposition follow similarly.

4. The results of $\$ \S 2$ and 3 can be summarized in the following.

THEOREM. Let $M$ be a linear subspace of countable codimension in a locally convex space $E$. If $E$ is w-barrelled, has property (C), or has property (S), then $M$ has the corresponding property when furnished with the relative topology.

5. The inheritance of the Mackey topology does not seem as certain as the inheritance of the before-mentioned properties. Our partial results are contained in the following

Proposition. Let $M$ be a linear subspace of countable codimension in a Mackey space E. Either of the following two sets of conditions is sufficient in order that $M$ be a Mackey space when furnished with the relative topology.

(a) $M$ is closed in $E$ and $E$ possesses property (S), or

(b) $M$ is dense in $E$, is separable, and $E$ possesses property (C).

Proof. If condition (a) is satisfied, then $M$ is a direct summand of $E$ by Proposition 2 of [4]. Since $E$ is a Mackey space, $M$ must then be a Mackey space.

If condition (b) is satisfied, $M^{\prime}$ can be canonically identified with $E^{\prime}$. Let $B$ be any $\sigma\left(E^{\prime}, M\right)$-compact subset of $E^{\prime}$. $B$ is $\sigma\left(E^{\prime}, M\right)$ bounded, and by Lemma 2 of this paper, $B$ is $\sigma\left(E^{\prime}, E\right)$-bounded, so $B$ is $\sigma\left(E^{\prime}, E\right)$-precompact. Suppose $\left\{f_{\alpha}\right\}_{\alpha \in D}$ is a $\sigma\left(E^{\prime}, E\right)$-Cauchy net in $B$. Since $B$ is $\sigma\left(E^{\prime}, M\right)$-compact, there exists $f \in B$ such that $\left\{f_{\alpha}\right\}_{\alpha \in D}$ is $\sigma\left(E^{\prime}, M\right)$-convergent to $f$. Suppose there were to exist $x_{0}$ in $E$ such that $\left\{f_{\alpha}\left(x_{0}\right)\right\}_{\alpha \in D}$ does not converge to $f\left(x_{0}\right)$. Then there exists $\epsilon>0$ such that the formula $\left|f_{\alpha}\left(x_{0}\right)-f\left(x_{0}\right)\right| \geqq \epsilon$ would be true for frequently many $\alpha$. Let $\left\{x_{n}: n \in \omega\right\}$ be a countable dense subset of $M$. By diagonalization, there would exist a sequence $\left\{\alpha_{j}\right\}_{j \in \omega}$ in $D$ such that the following statement would hold:

$\lim _{\boldsymbol{j}} f_{\alpha_{j}}\left(x_{n}\right)=f\left(x_{n}\right) \quad$ for $n \in \omega$, and $\left|f_{\alpha_{j}}\left(x_{0}\right)-f\left(x_{0}\right)\right| \geqq \epsilon$ for $j \in \omega$. 
If $\left\{f_{\alpha_{j}}: j \in \omega\right\}$ were infinite, since it is $\sigma\left(E^{\prime}, E\right)$-bounded, then property (C) implies that it would have a $\sigma\left(E^{\prime}, E\right)$-accumulation point $g \in E^{\prime}$ such that the formulas

$$
g\left(x_{n}\right)=f\left(x_{n}\right) \quad \text { for } n \in \omega, \quad \text { and } \quad\left|g\left(x_{0}\right)-f\left(x_{0}\right)\right| \geqq \epsilon
$$

hold true. If $\left\{f_{\alpha_{j}}: j \in \omega\right\}$ were finite, there would exist $k \in \omega$ such that the formulas

$$
f_{\alpha_{k}}\left(x_{n}\right)=f\left(x_{n}\right) \quad \text { for } n \in \omega, \text { and } \quad\left|f_{\alpha_{k}}\left(x_{0}\right)-f\left(x_{0}\right)\right| \geqq \epsilon
$$

held true. Then let $g=f_{\alpha_{k}}$. In either case, $g-f$ would be a continuous linear functional on $E$ which vanished on a dense subset at a dense subset of $E$. Then $g-f$ would be identically zero. This would contradict the inequality $\left|g\left(x_{0}\right)-f\left(x_{0}\right)\right| \geqq \epsilon$. Then the $\sigma\left(E^{\prime}, M\right)$-limit of every $\sigma\left(E^{\prime}, E\right)$-Cauchy net of $B$ is also its $\sigma\left(E^{\prime}, E\right)$-limit. $B$ has now been shown to be $\sigma\left(E^{\prime}, E\right)$-compact. $B$ is therefore equicontinuous with respect to $E$ and is all-the-more-so equicontinuous with respect to $M$. Then $M$ is a Mackey space.

Corollary. Let $M$ be a linear subspace of countable codimension of a Mackey space E. If $M$ is separable and $E$ has property (C), then $M$ is a Mackey space when furnished with the relative topology.

REMARK. The following example shows that condition (b) of Proposition 5 cannot be weakened to the condition that $M$ be a dense linear subspace of a Mackey space. Let $E$ be any quasi-reflexive Banach space (see [1]) such that $J(E)$ is of codimension 1 in $E^{\prime \prime}$, where $J$ is the canonical imbedding of $E$ into $E^{\prime \prime} . J(E)$ is $\sigma\left(E^{\prime \prime}, E^{\prime}\right)$ dense in $E^{\prime \prime}$, so $J(E)$ is $\tau\left(E^{\prime \prime}, E^{\prime}\right)$-dense in $E$. $J(E)$ is complete in $\tau\left(J(E), E^{\prime}\right)$, but is not complete in the relative $\tau\left(E^{\prime \prime}, E^{\prime}\right)$-topology. The two topologies must then be different, so $J(E)$ is not a Mackey space with the relative $\tau\left(E^{\prime \prime}, E^{\prime}\right)$-topology.

6. The following examples demonstrate the independence of the several properties discussed in this paper. Consider the vector space $m$ of all bounded sequences with the topology $J=\tau\left(m, l_{1}\right)$.

Proposition. $(m, J)$ is a Mackey space with property (S) but not property $(\mathrm{C})$.

Proof. $l_{1}$ is $\sigma\left(l_{1}, m\right)$-sequentially complete (see [2, Chapter IV, Table IV, A, p. 374]) so $(m, J)$ has property $(\mathrm{S}) .(m, J)$ is certainly a Mackey space. Let $B=\left\{e_{n}: n \in \omega\right\}$ be the canonical Schauder basis of $l_{1} ; e_{n}$ is the sequence of all zeros except the $n$th term, which is $1 . B$ is $\sigma\left(l_{1}, m\right)$-bounded, but has no $\sigma\left(l_{1}, m\right)$-accumulation point in $l_{1}$. 
For suppose $y \in l_{1}$. There exists a sequence $\phi \in m$ of the form $\phi$ $=(0, \cdots, 0,2,2 \cdots)$ such that $|\langle\phi, y\rangle|\left\langle 1\right.$. Thus $\left\langle\phi, e_{n}-y\right\rangle$ $=\left\langle\phi, e_{n}\right\rangle-\langle\phi, y\rangle>1$ holds true for $n$ big enough that $\left\langle\phi, e_{n}\right\rangle=2$. Then $e_{n}-y \in\{\phi\}^{\circ}$ for at most finitely many $n \in \omega$. Therefore $y$ is not a $\sigma\left(l_{1}, m\right)$-accumulation point of $B$.

We now show that there exist $\omega$-barrelled spaces which are not barrelled. Then the results of this paper do not reduce to those of [3]. Let $E$ be a vector space with uncountable algebraic (Hamel) basis $B$. Let $F$ be the subspace of the algebraic dual $E^{*}$ of $E$ consisting of $f \in E^{*}$ such that $f(x) \neq 0$ for at most countably many $x \in B$. Then $\langle E, F\rangle$ is a dual pairing separating points. Let $J_{0}$ be the topology on $E$ of uniform convergence on countable $\sigma(F, E)$-bounded subsets of $F$. It is true that $J_{0}$ is compatible with the dual system $\langle E, F\rangle$. First, it is clear that $J_{0} \supset \sigma(E, F)$. On the other hand, let $f_{0}$ be a $J_{0}$-continuous element of $E^{*}$. There must then exist a countable, $\sigma(F, E)$-bounded subset $A \subset F$ such that $\left|f_{0}(e)\right| \leqq 1$ for every $e \in A^{\circ}$. Let $B_{1}$ $=\{x \in B: f(x) \neq 0$ for some $f \in A\} . B_{1}$ is necessarily countable. If $x \in B \sim B_{1}, \lambda x \in A^{\circ}$ for all scalars $\lambda$. Then $\left|f_{0}(\lambda x)\right| \leqq 1$ for all scalars $\lambda$, and we deduce that $f_{0}(x)=0$. Then $f_{0} \in F$. We have shown that $\Xi_{0} \subset \tau(E, F)$. Since the polars of countable $\sigma(F, E)$-bounded subsets of $F$ are $J_{0}$-neighborhoods of 0 , they are also $\tau(E, F)$-neighborhoods of 0 .

Proposition. $(E, \tau(E, F))$ is an w-barrelled Mackey space which is not barrelled.

Proof. We now only need to prove the assertion that $(E, \tau(E, F))$ is not a barrelled space. Let $\mathcal{F}$ be the family of finite subsets of $B$. For each $C \in \mathcal{F}$ let $f_{C}$ be the functional in $F$ such that $f_{C}(x)=1$ for $x \in C$, $f_{C}(x)=0$ for $x \in B \sim C$. The set $A=\left\{f_{C}: C \in F\right\}$ is a $\sigma(F, E)$-bounded subset of $F$. The functional $g \in E^{*}$ such that $g(x)=1$ for all $x \in B$ is the $\sigma\left(E^{*}, E\right)$-limit of a net in $A$. $A$ cannot be equicontinuous then, for otherwise $g$ would be $\tau(E, F)$-continuous, which it is not.

Proposition. $\left(E, J_{0}\right)$ is an w-barrelled space which is not a Mackey space.

Proof. Let $V$ be the set of elements $y$ of $E$ such that if we write $y=\sum_{x \in B} \lambda_{x} x$, then $\left|\lambda_{x}\right| \leqq 1$ for all $x \in B$. $V$ is a barrel in $E$. Let $U$ be any 3 -neighborhood of 0 . There exists a countable subset $B_{0} \subset B$ such that $\lambda x \in U$ for all scalars $\lambda$ and all $x \in B \sim B_{0}$. Let $f$ be any element of $E^{*} \sim F$. Then there exist uncountably many elements $x$ of $B \sim B_{0}$ such that $f(x) \neq 0$. Then there exists an $\epsilon>0$ such that for infinitely many elements $x$ of $B \sim B_{0}$, it is true that $|f(x)| \geqq \epsilon$. It follows that $f$ is unbounded on $V \cap U . V$ is therefore a $\tau(E, F)$-neighborhood of 0 . It 
is also true that $V$ contains no line. On the other hand, since $B$ is uncountable, the polar of any countable subset of $F$ must contain a line. Therefore, $V$ is not a $J_{0}$-neighborhood of 0 , and $J_{0}$ is not $\tau(E, F)$.

Proposition. Let $E$ be any infinite dimensional vector space with algebraic dual $E^{*}$. Then $\left(E, \sigma\left(E, \pi E^{*}\right)\right)$ has property $(\mathrm{C})$, but is not $\omega$ barrelled.

Proof. Every $\sigma\left(E^{*}, E\right)$ bounded subset of $E^{*}$ is relatively $\sigma\left(E^{*}, E\right)$ compact, so $\left(E, \sigma\left(E, E^{*}\right)\right)$ has property (C). Let $B$ be any Hamel basis of $E$. Let $B_{0}$ be a countably infinite subset of $B$. For $x \in B_{0}$, let $f_{x}$ be that linear functional in $E^{*}$ such that

$$
f_{x}(x)=1, f_{x}(y)=0 \text { for } y \in B \sim\{x\} .
$$

The set $A=\left\{f_{x}: x \in B_{0}\right\}$ is a countable, $\sigma\left(E^{*}, E\right)$-bounded subset of $E^{*} . A^{\circ}$ contains no finite-codimensional subspace of $E$. Then $A$ is not equicontinuous and $\left(E, \sigma\left(E, E^{*}\right)\right)$ is not $\omega$-barrelled.

As a final example, let $c_{0}$ be the normed space of real sequences convergent to zero. Then $\left(l_{1}, \tau\left(l_{1}, c_{0}\right)\right)$ is an example of a Mackey space which does not have property (S).

7. It is apparent that if $E$ is a locally convex space, then $E$ is barrelled implies $E$ is $\omega$-barrelled; $E$ is $\omega$-barrelled implies that $E$ has property (C); $E$ has property (C) implies that $E$ has property (S). It can be shown that, moreover, if $E$ is metrizable, then $E$ has property (S) implies that $E$ is barrelled. The examples in $\$ 7$ show, however, that if $E$ is not metrizable, even though $E$ might be a Mackey space, $E$ has property (S) does not imply that $E$ has property (C); $E$ is $\omega$ barrelled does not imply that $E$ is barrelled. If $E$ is not a Mackey space then $E$ has property (C) does not imply that $E$ is $\omega$-barrelled. The authors do not know if a Mackey space with property (C) must be $\omega$-barrelled.

The authors are very grateful to the referee for his helpful suggestions.

\section{REFERENCES}

1. P. Civin and B. Yood, Quasi-reflexive spaces, Proc. Amer. Math. Soc. 8 (1957), 906-911. MR 19, 756.

2. N. Dunford and J. T. Schwartz, Linear operators. I: General theory, Pure and Appl. Math., vol. 7, Interscience, New York, 1958. MR 22 \#8302.

3. J. Horváth, Topological vector spaces and distributions. Vol. I, Addison-Wesley, Reading, Mass., 1966. MR 34 \#4863.

4. S. Saxon and M. Levin, Every countable-codimensional subspace of a barrelled space is barrelled, Proc. Amer. Math. Soc. 29 (1971), 91-96.

University of Florida, Gainesville, Florida 32601

Florida State University, Tallahassee, Florida 32306 